\title{
Prospección y datación de la fecha de colonización del área del Río Hollemberg, provincia de Última Esperanza, el punto más septentrional de presencia de Castor canadensis (Castoridae) en Sudamérica
}

\author{
Survey and tree-ring dating of colonized Hollemberg River Area, \\ Última Esperanza Province, the northernmost point of \\ Castor canadensis (Castoridae) presence in South America
}

Giorgia Graells ${ }^{1}$, Derek Corcoran² \& Juan Carlos Aravena ${ }^{1}$

\section{Resumen}

Castor canadensis es una especie invasora en el extremo sur de Sudamérica que, a partir de su introducción en el año 1946, se ha dispersado desde la isla Tierra del Fuego a algunas áreas del archipiélago fueguino y la porción más austral del continente americano, llegando incluso a la provincia de Última Esperanza el año 2013, en donde un ejemplar de la especie fue identificado y cazado por personal de Servicio Agrícola y Ganadero en el río Hollemberg (5154'31,6"' S, 72²6'3,9' W).

Para determinar la fecha real de llegada se realizó una prospección en las lagunas alimentadoras del río Hollemberg y una datación según técnicas dendrocronológicas de la fecha de corte del material leñoso usado por el castor capturado en el área.

La prospección no indicó nuevos sitios de presencia de castor en el área y sólo se encontró un árbol de la especie Nothofagus antárctica talado por castor. La datación dendrocronológica permitió estimar que el año de corte correspondió al 2011, determinando una diferencia entre la datación dendrocronológica y el avistamiento de castor de sólo dos años. Este breve período se podría explicar por el hecho de que en el área de estudio existe un mayor flujo de personas, asociadas a actividades de pesca deportiva, en relación con otras zonas que pueden estar siendo utilizadas como rutas de dispersión del castor y no han sido detectadas.

Palabras clave:

dendrocronología, castor, río Hollemberg, dispersión.

\begin{abstract}
Castor canadensis is an invasive species in Southern South America, which since 1946, the time of their introduction, was dispersed from Tierra del Fuego Island through some areas of Fueguian Archipelago and the southernmost portion of the American Continent. Beavers had arrived even to última Esperanza Province at 2013, where one specimen was identified and hunted by personnel of Servicio Agricola y Ganadero in the Hollemberg River (51 ${ }^{\circ} 54^{\prime} 31.6$ ' S, 72²6'3.9' W).
\end{abstract}

To determine the real time of the beaver's arrival to the area, we surveyed the feeding lagoons of the Hollemberg River, and the wood material used by the traped beaver was dated using dendrochronological techniques.

The survey did not show new places of beaver presence and we found just one small Nothofagus antarctica tree cut by the beaver. The tree-ring dating indicates that the date of cutting was 2011, with a two years of difference between tree-ring dating and the date people found that beaver. The short time difference indicates that Hollemberg River has more people visiting the area than other places that could be routes of dispersion of beavers, not being detected.

\footnotetext{
1 Universidad de Magallanes, Punta Arenas, Chile. Proyecto del que se deriva: Fondecyt 1130381. gygraell@gmail.com $\bowtie$

2 University of Missouri, Columbia MO, USA.
} 


\section{Key words:}

dendrochronology, beaver, Hollemberg River, dispersal.

\section{INTRODUCCIÓN}

El castor norteamericano (Castor canadensis, Kuhl 1820) es una especie invasiva en la Región de Magallanes y Antártica Chilena y es considerada una especie perjudicial o dañina por la ley $\mathrm{N}^{\circ} 19.473 / 96$ y su reglamento D.S. 05/98 y sus modificaciones, ambos del Ministerio de Agricultura. Esta clasificación se encuentra determinada por los daños que esta especie causa sobre el bosque magallánico. Entre ellos se destaca su capacidad de modificar el hábitat mediante el corte de árboles del género Nothofagus hasta una distancia superior a los $20 \mathrm{~m}$ desde el borde de la represa (Anderson et al. 2009), y la construcción de diques y castoreras (Muller-Schwarze \& Sun, 2003), que inundan extensas áreas que pueden alcanzar hasta $1.5 \mathrm{~m}$ de profundidad y $100 \mathrm{~m}$ de largo (Baldini et al. 2008). Estas alteraciones provocan dificultades para la regeneración del bosque que son moduladas por cambios en la comunidades vegetales y en los patrones de sucesión ecológica en los ecosistemas forestales y acuáticos (Anderson et al. 2006; Martínez-Pastur et al. 2006).

Trabajos recientes indican que la introducción de esta especie en el Lago Fagnano, al sur de Tierra del Fuego, fue realizada mediante la importación de sólo 20 castores y no 25 parejas, como se pensó originalmente (Pietrek \& Fasola, 2014). A partir de ese momento, en el año 1946, la especie se dispersó por toda la isla, islas aledañas como Dawson, Hoste y Navarino, e incluso en el continente, evidenciándose su llegada a la península de Brunswick ya en el año 1968 y su cruce hasta el seno Otway posterior al

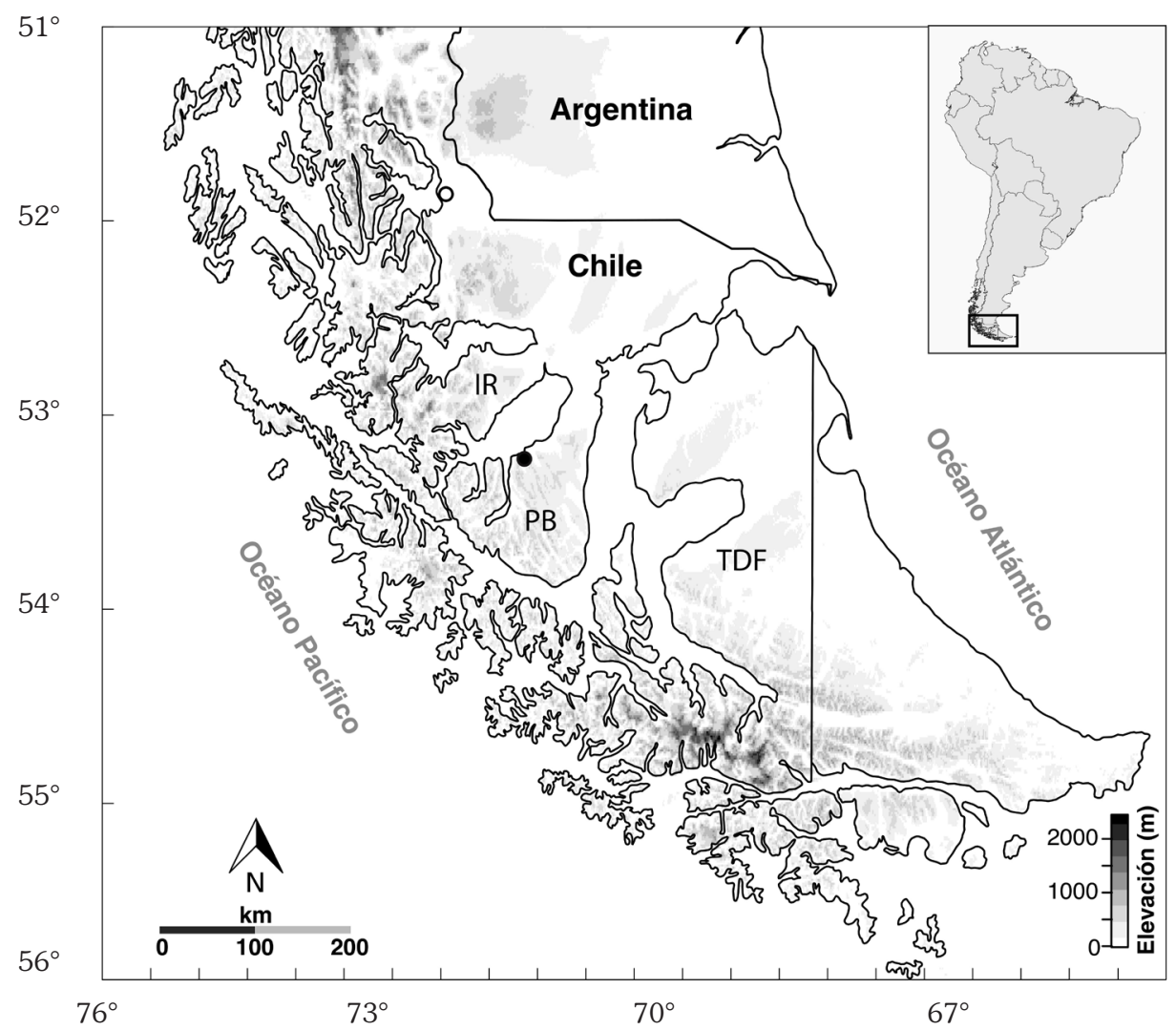

Fig. 1 Mapa general del área de estudio que comprende la Región de Magallanes y Antártica Chilena. TDF: Isla Tierra del Fuego, PB: Península de Brunswick, IR: Isla Riesco. El punto negro indica el sector del río Caleta en la Península de Brunswick, el punto blanco indica la ciudad de Puerto Natales. 
año 1986 (fechas obtenidas de Graells et al. 2015). El año 2013 los medios de prensa nacionales notificaron el descubrimiento de señales de la presencia del castor en el Río Hollemberg, en las cercanías de Puerto Natales (5143'34.540" N, $\left.72^{\circ} 30^{\prime} 22.543 " \mathrm{O}\right)$. El individuo correspondía a un ejemplar hembra adulta, sin signos de haber tenido crías (examen de necropsia SAG). Este nuevo registro de presencia, el más septentrional conocido hasta el momento, se encuentra a más de $110 \mathrm{Km}$ lineales del punto ubicado más al norte con registros de la especie en Río Verde y a $150 \mathrm{Km}$ lineales del punto más al norte datado con registro de presencia (río Caleta, península de Brunswick, Fig. 1), en el cual se estimó la llegada de castor el año 1986 (Graells et al. 2015).

En el trabajo de Corcoran (2014) se presenta una ruta potencial de movimiento del castor desde la isla de Tierra del Fuego al continente. Esta ruta pasa por la península de Brunswick y continúa hacia el norte del país, basándose en modelos de conectividad eléctrica y su adecuación biológica espacialmente explícita. Considerando que dicha ruta se encuentra a sólo $32 \mathrm{Km}$ del río Hollemberg, $15 \mathrm{Km}$ de laguna Diana y $20 \mathrm{Km}$ de la laguna Escondida, tomamos esta información como referencia para realizar una prospección de estos cuerpos de agua y procedimos a la datación del material leñoso afectado por castor encontrado en el río Hollemberg usando técnicas dendrocronológicas.

Dada la falta de información sobre la colonización del castor en la región de Magallanes, se hace necesario conocer la fecha de llegada real al sitio del río Hollemberg y realizar una prospección de los cuerpos de agua cercanos en busca de nuevos focos de invasión.

\section{MATERIALES Y MÉTODOS}

En noviembre del 2014 se realizó una prospección a pie del área del río Hollemberg que comprende desde la zona del lago Diana, laguna Escondida y lago Balmaceda (costa sur) hasta su desembocadura en la Bahía Desengaño, en el Golfo Almirante Montt, comuna de Puerto Natales (Fig. 2). Se desarrolló una búsqueda intensiva de señales de la presencia de castor norteamericano, como la construcción de represas, castoreras o cortes de árboles abarcando un área prospectada de aproximadamente $120 \mathrm{Km}^{2}$.

En el sitio en donde se encontraron señales

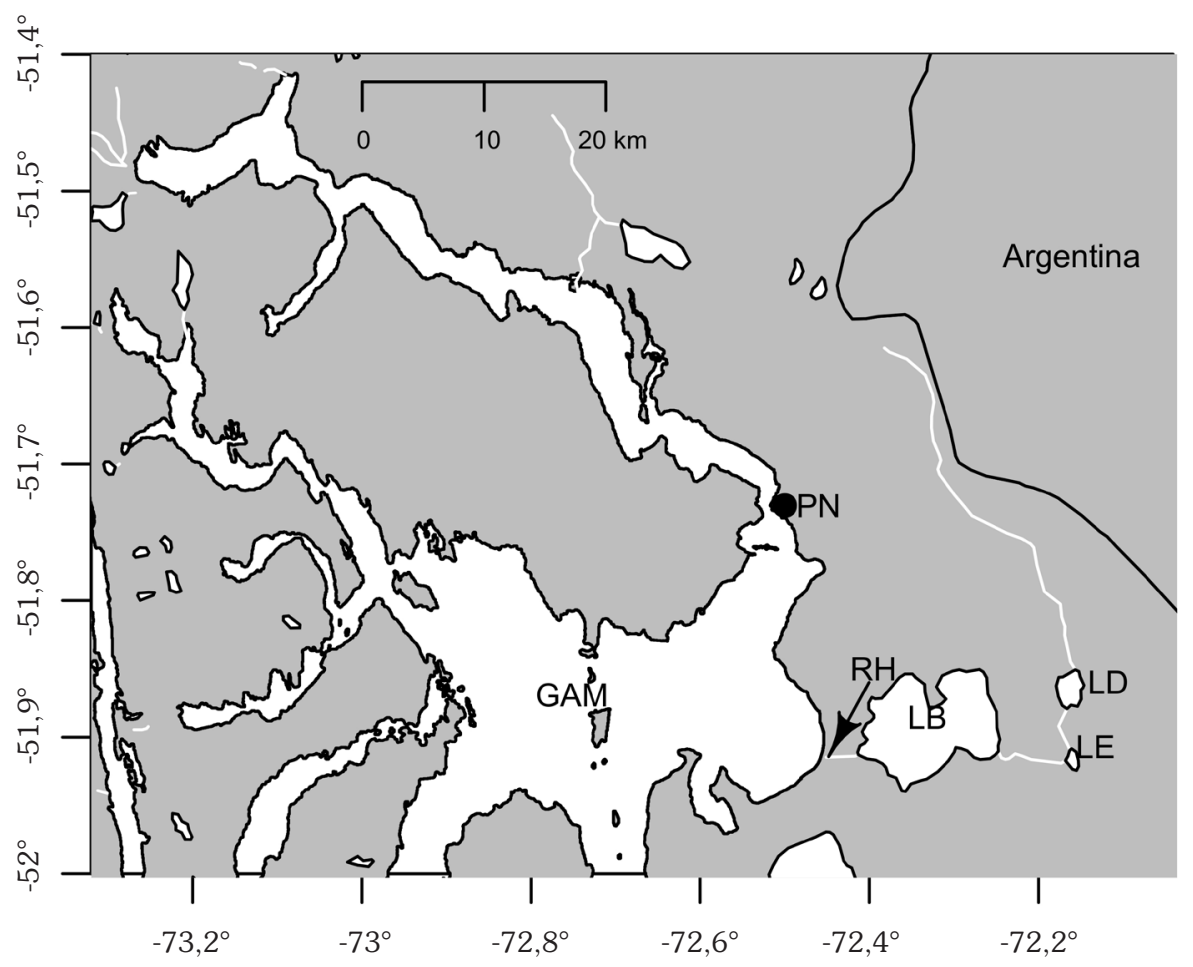

Fig. 2. Detalle del área de estudio situada en la provincia de Última Esperanza, Chile. PN: Puerto Natales, GAM: Golfo Almirante Montt, RH: río Hollemberg, LB: Lago Balmaceda, LD: Laguna Diana, LE: Laguna Escondida. 
de castor se registraron las coordenadas geográficas con un GPS Garmin eTrex. Se tomaron muestras dendrocronológicas de material leñoso arbóreo sin crecimiento posterior al corte de castor, los que permitieron obtener serie de anillos lo suficientemente largas para trabajar cronologías (Stokes \& Smiley, 1968). Las muestras fueron tomadas mediante barrenos Haglof en árboles vivos aledaños al sitio con evidencia de castor, mientras que en el caso de árboles cortados se obtuvieron rodelas mediante una sierra manual.

Las muestras fueron llevadas al Laboratorio de Botánica del Instituto de la Patagonia, Universidad de Magallanes, en donde fueron procesadas. Las muestras de árboles vivos fueron montadas en molduras de madera (Stokes \& Smiley, 1968; Phipps, 1985) y fijadas mediante pegamento soluble al agua para mantener la posición de la muestra según el alineamiento de las células. Se mantuvieron con cinta adhesiva de papel para evitar su levantamiento mientras secaba el pegamento. Tanto las muestras de los árboles vivos como las rodelas de las ramas cortadas fueron preparadas utilizando una lijadora eléctrica con lijas en un rango de 100 a 800 de grano (Pilcher, 1990).

La medición del ancho de los anillos de las muestras procesadas se realizó con una lupa estereoscópica usando un aumento de $15 x$ y se registró de forma digital con un equipo VELMEX. Las series de anchos de los anillos de árboles vivos y muertos fueron analizados mediante el programa COFECHA (Holmes, 1983). Se compararon los patrones de crecimiento de árboles vivos (fechas conocidas) con los del árbol cortado por castor y se determinó la fecha de muerte del mismo por cofechado.

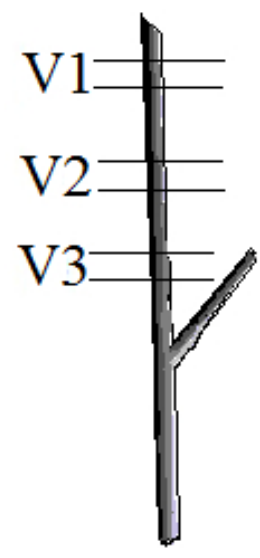

Fig. 3. Esquema de la toma de muestras del árbol encontrado cortado por castor en el río Hollemberg. V1, V2 y V3 indican las muestras del árbol viejo a partir de cortes transversales realizado para el posterior procesamiento. Las tres muestras fueron tomadas de la misma rama del árbol cortado.

\section{RESULTADOS}

En el recorrido de $20 \mathrm{Km}$ de prospección (Fig. 3) sólo se logró identificar señales de presencia de castor en el área del río Hollemberg, determinado anteriormente por personal del Servicio Agrícola y Ganadero y reportado por los medios de comunicación. En el sitio se identificaron dos especies vegetales utilizadas como recurso leñoso por castor: Escallonia virgata (Ruiz et Pavón) Pers. y Nothofagus antárctica, (G. Forst (Oerst)). Se obtuvo tres muestras (pseudoréplicas: HOL1V1, HOL1V2, HOL1V3) de un árbol cortado por castor para datación y 24 muestras dobles de árboles vivos para cronología.

Para el cofechado de árboles vivos se utilizaron sólo12 muestras, que fueron aquellas que presentaron un índice de correlación mayor a 0,393 (Tabla 1). La datación dendrocronológica de las muestras del río Hollemberg cofechó tres fechas estadísticamente posibles de corte del ár-

Tabla 1. Resumen de las muestras dendrocrológicas para el sitio del río Hollemberg.

\begin{tabular}{ccccc}
\hline $\begin{array}{c}\text { Número de } \\
\text { muestras de árboles } \\
\text { vivos }\end{array}$ & $\begin{array}{c}\text { Correlación con } \\
\text { la serie maestra }\end{array}$ & $\begin{array}{c}\text { Número de árboles vivos } \\
\text { usados para cofechado }\end{array}$ & $\begin{array}{c}\text { Número de muestras } \\
\text { de árboles cortados }\end{array}$ & $\begin{array}{c}\text { Número de réplicas del } \\
\text { árbol cortado por castor }\end{array}$ \\
\hline 24 & 0,393 & 9 & 1 & 3 \\
\hline
\end{tabular}

Tabla 2. Información de las tres muestras dobles tomadas para el árbol cortado por castor en el río Hollemberg.

\begin{tabular}{ccccc}
\hline Muestra & Año de inicio & Correlación & Edad & Año de corte \\
\hline HOL1V1 & 1992 & 0,425 & 19 & 2011 \\
HOL1V2 & 1992 & 0,23 & 19 & 2011 \\
HOL1V3 & 1992 & 0,405 & 19 & 2011 \\
\hline
\end{tabular}


bol por castor: 1958, 1976 y 2011, todas con una alta correlación en las muestras con la serie maestra de árboles vivos del sector. Se eligió el año 2011 como la fecha más parsimoniosa por cuanto la menor cantidad de años pasados desde su corte, que esta alternativa supone, concuerda con el estado de preservación del árbol cortado. Las muestras colectadas indican que el árbol encontrado en el río Hollemberg tendría 19 años de edad, habría nacido el año 1992 y muerto por el corte del castor el año 2011 (Tabla 2).

\section{DISCUSIÓN}

La ruta de migración del castor propuesta por Corcoran (2014) entregó una directriz del área a recorrer para determinar la posible presencia de castores al norte del río Canelo, en la península de Brunswick. Sin embargo, en nuestra prospección no fueron encontrados más rastros de la especie o de su paso entre el punto registrado en el río Hollemberg y el área de los cuerpos de agua definidos en este trabajo, lo que concuerda con información obtenida por el SAG en prospecciones anteriores.

Las tres fechas entregadas por la datación dendrocronológica: 1958, 1976 y 2011, poseen una alta correlación con la serie maestra. Dado que la muestra tenía sólo 19 años (simple conteo de anillos de crecimiento), se presenta una alta probabilidad de error en cuanto a la determinación de edad dentro de la serie maestra debido al pequeño tamaño de la muestra. Considerando que la tasa de descomposición $k$ de un tronco de $8 \mathrm{~cm}$ de diámetro de $N$. antártica es de 0,079 por año (cuociente biomasa/ año) (Frangi et al. 1997; Frangi et al. 2004), se determinó que la fecha de corte más parsimoniosa sería la del año 2011, sólo dos años antes de la fecha de su primer avistamiento.

El punto más al norte previamente datado por dendrocronología al del río Hollemberg corresponde al río Caleta, en la península de Brunswick, que indica que la colonización del castor ocurrió en 1986 (Graells et al. 2015). Entre ambos puntos de presencia fechada de castores existe una distancia de $150 \mathrm{Km}$ lineales y una diferencia de fechado de 25 años, lo cual implica que la migración de la especie entre ambos puntos (independiente de la ruta tomada) debió haber tomado mínimo 25 años. Sin embargo, el proceso migratorio cuenta con otros tres puntos no datados de presencia de castor confirmada por el Servicio Agrícola y Ganadero: río Canelo sin señales de presencia de castor, solo observación (Seremi de Agricultura de Magallanes y la Antártica Chilena, 2013), Estancia Entre Vientos con señales de construcción de represa y Estancia Río Verde con material leñoso cortado por castor, estos últimos ambos en la comuna de Río Verde (Nicolás Soto y Rodrigo Molina, comunicación personal).

Dado que los registros de castores marcados en Norteamérica muestran distancias migratorias de no más de $50 \mathrm{Km}$ lineales o $82 \mathrm{Km}$ por agua durante el período de dispersión (Beer, 1955), se sugiere que el avistamiento del castor solitario en el río Hollemberg podría no corresponder a un solo dispersante en el área, sino que a una oleada migratoria en etapas. Si se consideran los puntos de presencia confirmados y el posible desplazamiento de cada castor en un máximo de $50 \mathrm{Km}$, la Fig. 4 muestra las posibilidades de movimiento de distintos individuos, considerando cada punto de presencia como el centro de un radio de dispersión. La cercanía entre los radios de dispersión indica que el movimiento del castor por la región es posible y que los $150 \mathrm{Km}$ lineales fueron recorridos por distintos individuos que fueron dejando su descendencia, posiblemente en los puntos descritos.

A pesar de que se ha logrado identificar varios sitios de presencia de castor en la región de Magallanes y Antártica Chilena y se ha propuesto una ruta de dispersión de la especie bastante verosímil hacia el norte de nuestro de país, no se ha encontrado una población evidentemente establecida en el continente, con represas y castoreras activas, y con clara relación de continuidad como se observa en Tierra del Fuego, Navarino y Dawson. Sin embargo, no podemos dejar de mencionar que existe una falta de conocimiento en la distribución real de esta especie en la región, en particular en el área de fiordos y canales, en donde se presentan sitios no prospectados o zonas de difícil acceso que hacen que esta tarea sea difícil o de un muy alto costo para su realización. 


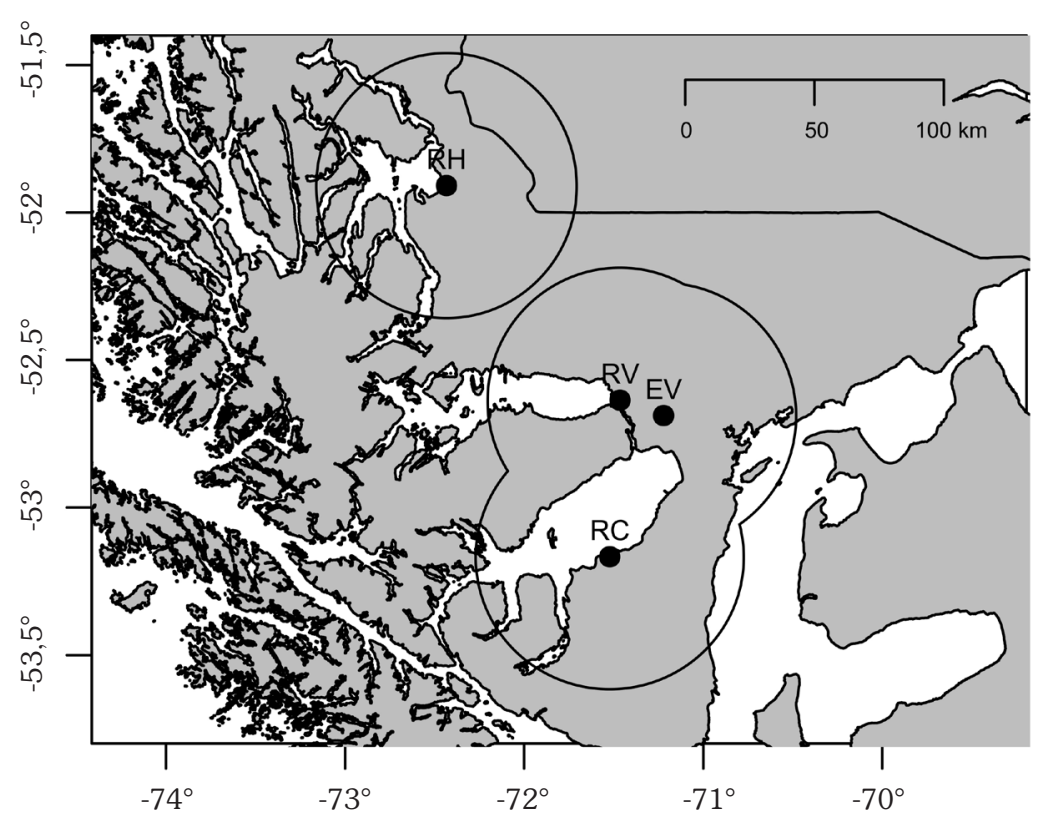

Fig. 4. Mapa del área continental de la invasión de C. canadensis. Los círculos indican el potencial radio de dispersión de un castor establecido en cada sitio de presencia confirmada (determinado por los puntos negros). $\mathrm{RH}$ : río Hollemberg, RV: sector de río verde, EV: estancia Entre Vientos, RC: río Caleta.

\section{CONCLUSIÓN}

El río Hollemberg presentó una situación particular de avistamiento de castor, en donde en un área aislada fue descubierto un ejemplar de castor por un pescador que conocía la especie y dio la alerta. La valoración de esta área para la pesca generó que sólo un individuo de la especie fuera suficiente para su identificación y erradicación. Sin embargo, la falta de estas condiciones en el resto de la región podría estar generando falta de información en cuanto a la presencia real del castor norteamericano en el sur de Sudamérica como así también sobre su ruta de dispersión.

\section{AGRADECIMIENTOS}

A Rafael Contreras de la Corporación Nacional Forestal (CONAF) de Puerto Natales, por la ayuda en el recorrido y reconocimiento del sitio de presencia de castor en el río Hollemberg, y a Inés Dussaillant por el apoyo en la toma de muestras.

\section{LITERATURA CITADA}

Anderson, C. B., Griffith, C. R., Rosemond, A. D., Rozzi, R., \& Dollenz, O. (2006). The effects of invasive North American beavers on riparian plant communities in Cape Horn, Chile: do exotic beavers engineer differently in sub-Antarctic ecosystems? Biological Conservation, 128, 467-474.

Anderson, C. B., Martinez-Pastur, G., Lencinas, M. V., Wallem, P. K., Moorman, M. C., \& Rosemond, A. D (2009). Do introduced North American beavers Castor canadensis engineer differently in southern South America? An overview with implications for restoration. Mammalian Review, 39, 33-52.

Baldini, A., Oltremari, J., \& Ramírez, M. (2008). Impacto del castor (Castor canadensis, Rodentia) en bosques de lenga (Nothofagus pumilio) de Tierra del Fuego, Chile. Bosque Valdivia, 29,162-169.

Beer, J. R. (1955) Movements of tagged beaver. Journal of Wildlife Management, 19, 492- 493.

Corcoran, D. (2014). Distribución potencial y possible ruta de migración de castor americano (Castor canadensis) en su rango invasivo utilizando el concepto de Nicho Hutchinsoniano. Tesis doctorado, Pontificia Universidad Católica de Chile, Santiago.

Frangi, J. L., Barrera, M. D., Puigdefábregas, J., Yapura, P. F., Arambarri, A. M., \& Richter, L. (2004). Ecología de los bosques de Tierra 
del Fuego. Ecología y Manejo de los Bosques de Argentina. Editorial de la Universidad Nacional de La Plata, La Plata.

Frangi, J. L., Richter, L. L., Barrera, M. D., \& Aloggia. M. (1997) Decomposition of Nothofagus fallen woody debris in forests of Tierra del Fuego, Argentina. Canadian Journal of Forest Research, 27, 10951102.

Graells, G., Corcoran, D., \& Aravena, J. C. (2015) Invasion of North American beaver (Castor canadensis) in the Province of Magallanes, Southern Chile. Comparison between dating sites through interviews with the local community and dendrochronology. Revista Chilena de Historia Natural, 88, 3-9.

Holmes, R. L. (1983) Computer-assisted quality control in tree-ring dating and measurement. Tree-Ring Bulletin, 43, 69-78.

Martínez Pastur, G., Lencinas, M. V., Escobar, J., Quiroga, P., Malmierca, L., \& Lizarralde, M. (2006). Understorey succession in Nothofagus forests in Tierra del Fuego (Argentina) affected by Castor canadensis. Applied Vegetation Science, 9, 143-154.

Muller-Schwarze, D., \& Sun, L. (2003). The Bea- ver: Natural History of a Wetlands Engineer. First edition. Comstock Publishing Associates, Ithaca, N.Y

Phipps, R. L. (1985). Collecting, preparing, crossdating, and measuring tree increment cores. US Department of the Interior, Geological Survey

Pietrek, A. G., \& Fasola, L. (2014). Origin and history of the beaver introduction in South America. Mastozoología Neotropical, 21, 355-359.

Pilcher, J. R. (1990). Sample preparation, cross-dating and measurement. En E. R. Cook \& L. A. Kairiukstis (Eds.). Methods in Dendrochronology: Applications in the Environmental Sciences. (pp. 40-50) Springer Science \& Business Media, Berlin.

Seremi de Agricultura de Magallanes y la Antártica Chilena (2013) Agronoticias. Boletin N46. Agosto 2013. Punta Arenas. URL: http://www.wobook.com/ WBIB63F8VB8t/Boletin-SEREMI-AGROMagallanes-Agosto-2013.html

Stokes, M. A., \& Smiley, T. L. (1968). Tree-Ring Dating. University of Chicago Press, Chicago. 
